\title{
Diversity of epidermal growth factor receptor-mediated activation of downstream molecules in human lung carcinomas
}

\author{
Shioto Suzuki ${ }^{1}$, Satoshi Igarashi ${ }^{1}$, Mitsuhiko Hanawa ${ }^{1}$, Hirochika Matsubara ${ }^{2}$, Akishi Ooi $^{3}$ \\ and Yoh Dobashi ${ }^{1}$ \\ ${ }^{1}$ Department of Pathology, Interdisciplinary Graduate School of Medicine and Engineering, University \\ of Yamanashi, Yamanashi, Japan; ${ }^{2}$ Second Department of Surgery, Faculty of Medicine, University of \\ Yamanashi, Yamanashi, Japan and ${ }^{3}$ Department of Molecular and Cellular Pathology, Kanazawa University \\ Graduate School of Medical Science, Ishikawa, Japan
}

\begin{abstract}
The correlations among epidermal growth factor receptor (EGFR) gene amplification, gene mutation, overexpression/phosphorylation of EGFR protein and activation of its downstream molecules, signal transducers and activators of transcription 3 (Stat-3), Akt and extracellular signal-related protein kinase $1 / 2$ (Erk1/2) were investigated in 28 cases of human lung carcinomas. In five cases of carcinomas with EGFR amplification, EGFR expression and phosphorylation levels were higher than other cases, and Stat-3 was activated in all five cases. Point mutations in the tyrosine kinase domain of EGFR were detected in five cases, one of which was also associated with gene amplification. In these five cases, both EGFR expression and phosphorylation were enhanced, and Akt was activated in four cases. In the remaining 19 cases, EGFR protein expression was upregulated in eight cases and phosphorylated in four cases, but neither EGFR nor phosphorylated-EGFR expression levels specifically correlated with activation of particular downstream molecules. In general, either Stat-3 or Akt, but not both, was activated reciprocally and complementarily to each other, as indicated by their phosphorylation. However, Erk1/2 was activated regardless of the status of Stat-3, Akt or EGFR proteins. The current data suggest that persistent Stat-3 activation may be a critical event downstream of EGFR that has been overexpressed by gene amplification. In contrast, tumor cells harboring the EGFR mutation may persistently activate a cascade via Akt. Finally, in the majority of cases that have no aberration of the EGFR, its downstream molecules function in reciprocal and/or complementary manner in the maintenance and/or progression of carcinomas. These overall results could provide novel insights into potential chemotherapeutic regimens for lung carcinomas, such as inhibitors of Stat-3, Akt and Erk1/2. Modern Pathology (2006) 19, 986-998. doi:10.1038/modpathol.3800619; published online 28 April 2006
\end{abstract}

Keywords: EGFR; gene aberrations; Stat-3; Akt; lung carcinoma

Current surgical and chemotherapeutic intervention for lung carcinomas has prolonged survival of patients in advanced stages; however, the overall prognosis still remains unsatisfactory with a cure rate of less than $15 \% .^{1}$ Several new strategies for targeting specific molecules have been developed, aiming, in particular, at the inhibition of activated tyrosine kinase. ${ }^{2-4}$ This molecular targeting therapy was based on the assumption that the targeted

Correspondence: Dr Y Dobashi, MD, PhD, Department of Pathology, Interdisciplinary Graduate School of Medicine and Engineering, University of Yamanashi, 1110 Shimokato, Chuo, Yamanashi 409-3898, Japan.

E-mail: ydobashi@yamanashi.ac.jp

Received 8 March 2006; revised and accepted 5 April 2006; published online 28 April 2006 molecule is overexpressed, frequently by gene amplification and critically involved in tumors. In this line, analysis of HER2 gene amplification can be used to determine the response of breast cancer to the anti-HER2 therapy, Trastuzumab. Epidermal growth factor receptor (EGFR) has also been the target of cancer therapies owing to its critical role and for its frequent overexpression in many epithelial cancers, including non-small-cell lung cancer (NSCLC). ${ }^{2,3,5,6}$ Our previous study showed that EGFR protein was overexpressed in $34 \%$ of NSCLC, and gene amplification was observed in $74 \%$ of EGFR-overexpressing tumors. ${ }^{5}$ Nowadays, several EGFR-targeted therapies are in clinical use, including anti-EGFR monoclonal antibody cetuximab, as well as the tyrosine kinase inhibitors (TKIs), gefitinib and erlotinib., ${ }^{2,7}$ 
Gefitinib has shown dramatic clinical responses in about $10-19 \%$ of NSCLC that had been refractory to conventional chemotherapy. ${ }^{3,8}$ Most of these cases were revealed to have mutations in the kinase domain of EGFR gene. ${ }^{7,9-12}$ The response to gefitinib has been reported to be unaffected by EGFR expression or EGFR amplification. ${ }^{13-15}$ Consistently, gefitinib was less effective against squamous cell carcinoma (SCC) than adenocarcinoma (AC), despite the fact that SCC showed higher levels of EGFR expression, as it showed lower frequency of EGFR mutation. ${ }^{13}$ However, other studies have reported that EGFR amplification is associated with sensitivity to gefitinib/erlotinib and with better survival. ${ }^{16-19}$

Upon ligand binding, EGFR undergoes autophosphorylation and transduces signals through several cascades mediated by adaptors that bind to Cterminal phosphotyrosines and recruit proteins involved in downstream signaling events. Those cascades involve signal transducer and activator of transcription (STAT), phosphatidylinositol 3'-kinase (PI3K)/Akt and Ras/Raf/extracellular signal-regulated kinase 1/2 (Erk1/2) pathways. ${ }^{3,6,13,15,20,21}$

STATs are transcriptional factors that are activated through phosphotyrosine residues of EGFR to modulate cell proliferation and differentiation. ${ }^{21,22}$ Phosphorylation of STAT leads to its activation and translocation to the nucleus, where it binds to DNAresponse elements. ${ }^{21}$ Constitutive activation of STATs, in particular, Stat-3 is found in human malignant tumors and cancer cell lines, including carcinomas of the head and neck, lungs and others. ${ }^{23-25}$

Akt is phosphorylated and activated by EGF through PI3-kinase and plays a critical role in various cellular actions, including glucose metabolism, DNA synthesis and antiapoptotic signaling. ${ }^{26,27}$ In addition, constitutively activated Akt can oncogenically transform cells and it has been reported to be hyperphosphorylated in many cases of NSCLC, although the frequency varies from 60 to $90 \%$ depending on the reports. ${ }^{26,28}$

Erk1/2 was originally shown to be activated by a variety of mitogenic agents, and is a ubiquitous component of signal transduction pathways regulating cell proliferation, survival and transformation. $^{20,29}$ Constitutive activation of Erk1/2 was observed in cultured cancer cells of the kidney, colon and the lungs as well as in $30 \%$ of NSCLC cases. ${ }^{29}$

Although these three cascades definitely play critical roles in EGFR signal transduction, the correlation between aberrations in EGFR and the activation of specific cascades in human tumors has not been elucidated. In fact, our previous study on soft tissue sarcomas showed that even in the cases with EGFR amplification, Erk1/2 was not always activated. ${ }^{30}$ One report has shown that in cell lines stably transfected with mutant EGFRs, phosphorylation of both Akt and STAT is induced by EGF stimulation. ${ }^{12}$ If this is the case, enhanced Akt and STAT signaling could be targets of therapeutic intervention and therefore, cells expressing mutant
EGFRs may be more sensitive to the combined pharmacological inhibition of Akt or STAT with gefitinib. However, responsiveness against gefitinib therapy is not uniform among tumors harboring EGFR mutations. There were exceptional cases of tumors that show expression of wild-type EGFR, but are nevertheless sensitive for gefitinib, and there are cases that express Gefitinib-resistant EGFR mutants. ${ }^{3,7,18,31,32}$ Furthermore, as the majority of the remaining cases express wild-type EGFR, we need to elucidate the details of the EGFR-activated cascades in order to determine the chemotherapeutic regimen. Clarification of these complicated issues will require comprehensive analyses to investigate whether the particular status of EGFR, that is, the presence of mutations or gene amplification, correlate with activation of specific downstream molecules. These results will ultimately determine the selection of chemotherapeutic agents and potential beneficial effects in each case.

For this purpose, we undertook a comprehensive analysis of EGFR aberrations and activation of EGFR-mediated signaling cascades in NSCLC in order to provide a rationale for the application of novel 'tailored' regimens of chemotherapy.

\section{Materials and methods}

\section{Tissue Samples and Histological Classification}

For this study, 28 cases of archival tissue samples embedded in paraffin and fresh frozen tissues of surgically resected specimens from patients with Stage I-IIIA NSCLC, together with their adjacent non-neoplastic lung tissues were utilized. All samples were obtained from the University of Yamanashi Hospital between 1995 and 2005 under protocols approved by the Institutional Human Tissue Utilization Committee (Project no. 45). Specimens include 17 cases of AC, 10 cases of SCC and one case of large cell carcinoma (LC). AC was further subclassified into 15 cases of 'adenocarcinoma of mixed subtype', one case of 'papillary carcinoma' and one case of 'solid adenocarcinoma with mucin'. ${ }^{33}$ Tissues from two patients with ruptured bulla were used as control samples from nontumor patients. Expression of EGFR protein by immunohistochemistry (IHC) and gene amplification by fluorescence in situ hybridization analysis had already been examined in these cases and described previously. ${ }^{5}$ Profiles of these 28 cases examined in the present study are summarized in Table 1 . At the time of surgery, none of the patients had received any treatment for lung cancer, including treatment with TKIs.

\section{Immunohistochemistry}

All tissue samples were routinely processed and serial sections of $3.5 \mu \mathrm{m}$ in thickness were used for 
Table 1 Results of immunohistochemistry and FISH analysis for 28 cases (Suzuki et al ${ }^{5}$ )

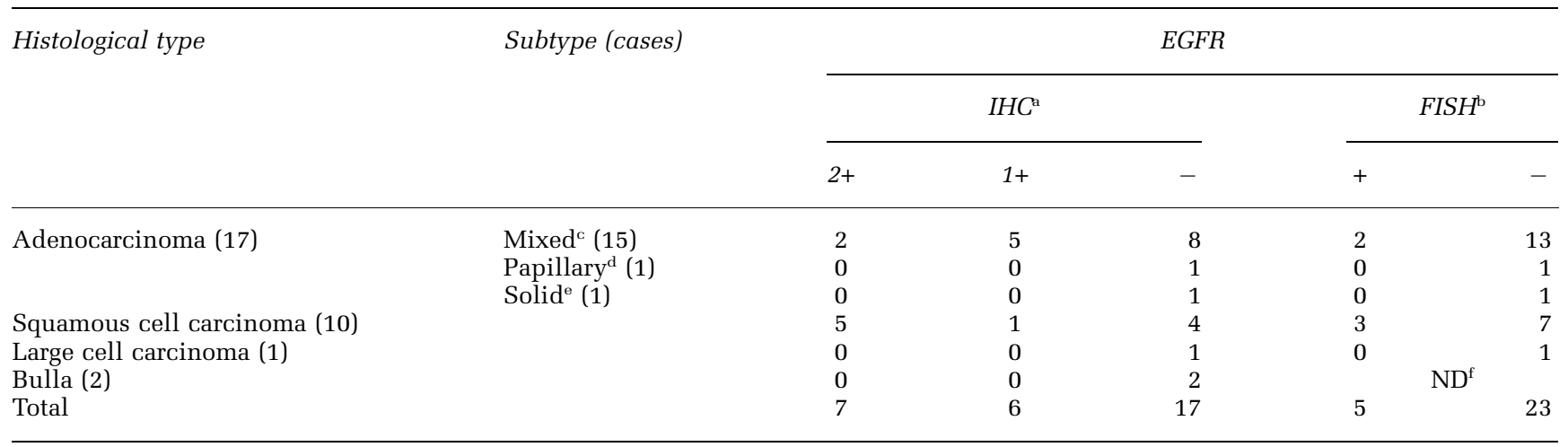

${ }^{\mathrm{a}} \mathrm{IHC}$, immunohistochemistry.

${ }^{\mathrm{b}} \mathrm{FISH}$, fluorescence in situ hybridization analysis for gene amplilfication.

${ }^{\mathrm{C}}$ Mixed, adenocracinoma of the mixed subtype.

${ }^{\mathrm{d}}$ Papillary, papillary adenocarcinoma.

${ }^{\mathrm{e}}$ Solid, solid adenocarcinoma with mucin.

${ }^{\mathrm{f}} \mathrm{ND}$, not done.

IHC. Sections were autoclaved and immunostained with primary antibodies commercialized by Cell Signaling Technology (Beverly, MA, USA) at the following dilutions; antibody against phosphorylated form of EGFR (p-EGFR ${ }^{\text {Tyr1068 }}$ polyclonal) 1:50, p-Akt ${ }^{\text {Ser473 }}$ (monoclonal); 1:50, p-Stat3 $3^{\text {Tyr705 }}$ (polyclonal); 1:100, p-Erk1/2 ${ }^{\text {Thr202/Tyr204 }}$ (polyclonal) at 1:50 dilution. Antibodies were visualized by labeled streptoavidin-biotin peroxidase complex method (DAKO Japan, Kyoto, Japan). In the previous studies, the intensity of EGFR immunoreactivity was scored using a four-tier system that we and others have established: negative, no discernible staining; $1+$, definite cytoplasmic staining and/or equivocal discontinuous membrane staining; $2+$, unequivocal membrane staining with moderate intensity; $3+$, strong and complete membrane staining. ${ }^{5,34}$ For the other proteins, p-Stat-3, p-Akt and p-Erk1/2, the intensity of reactivity was scored as positive $(+)$ or negative $(-)$ : positive $(+)$, definite nuclear staining with higher intensity than that of lymphocytes or endothelial cells which occasionally showed faint cytoplasmic staining; negative $(-)$, no discernible staining or staining with lower intensity than those cells.

\section{Immunoblotting Analysis}

Fresh tissues were homogenized, sonicated in highsalt lysis buffer $(0.5 \%$ Nonidet P-40, $0.05 \%$ SDS, $50 \mathrm{mM}$ Tris-HCl (pH 7.6), 0.2 M NaCl, 5 mM EDTA, $50 \mathrm{mM} \mathrm{NaF}$ and proteinase inhibitors) and supernatant was taken as the final lysate. ${ }^{35}$ Equal amounts $(30 \mu \mathrm{g})$ of protein were applied for immunoblotting (IB). The same antibodies that were used for IHC were utilized for IB. Antibody against EGFR (monoclonal, Novocastra, Newcastle, UK) was used at a 1:200 dilution, anti-phospho-EGFR at 1:250 and the other antibodies against p-Stat-3, p-Akt and p-Erk1/
2 were used at the same dilutions as in IHC. In addition, an anti- $\beta$-actin antibody (monoclonal, Cytoskeleton Inc., Denver, CO, USA) was used at a 1:1500 dilution as a loading control. Colorization was performed with nitroblue tetrazolium and 5bromo-4-chloro-3-indol-phosphate (Promega, Madison, WI, USA). The data obtained by IB was scanned and incorporated into Image Gauge (version 3.2, Fujifilm, Tokyo, Japan) for densitometric quantification. The protein amounts were calculated relative to $\beta$ actin which was assigned an arbitrary level of '10', and the quantitated protein level was expressed as the 'protein index', which was the value obtained in tumor tissue divided by that in paired normal tissue. When the value in normal tissue was ' 0 ', the value in tumor tissue was automatically used as 'protein index'. The results were confirmed by three independent experiments. In this study, protein expression was interpreted as 'upregulated' (for EGFR expression) or 'activated' (for phosphorylation of EGFR, Stat-3, Akt and Erk1/2) when (i) detectable expression was observed only in tumor tissue or (ii) the protein amount of the tumor tissue was more than 1.5-fold higher than that of the paired normal tissue ('protein index'>1.5). ${ }^{29}$

\section{Statistical Analysis}

Correlations between the results of IHC, IB and clinicopathological factors were analyzed using the unpaired comparison $t$-test, $U$-test of MannWhitney or Kruskal-Wallis tests. Patients' survival was analyzed by the Kaplan-Meier method with Logrank test for univariate analysis.

\section{Nucleotide Sequence Analysis}

For mutational analysis of the EGFR kinase domain, DNA was extracted from fresh-frozen specimens in 
extraction buffer $(0.5 \%$ SDS, $50 \mathrm{mM}$ Tris-HCl $[\mathrm{pH}$ 7.4), $0.1 \mathrm{M} \mathrm{NaCl}, 1 \mathrm{mM}$ EDTA, $0.5 \mathrm{mg} / \mathrm{ml}$ of Proteinase K (Roche Diagnostics, Indianapolis, IN, USA)] followed by phenol-chloroform extraction. Fragments of exons 18-21 were amplified by polymerase chain reaction (PCR) using primer sequences and amplification conditions as described previously. ${ }^{10}$ Multiple, independent PCR amplicons were subjected to direct forward and reverse sequencing reactions with the same primers used for PCR and by ABI BigDye Terminator kit v3.1 (Applied Biosystems, Foster City, CA, USA). Sequencing reaction products were electrophoresed on an ABI 3700 genetic analyzer. Sequence variations were determined using Sequence software (Applied Biosystems) with the EGFR reference sequence (NM_005228.3, National Center for Biotechnology Information).

\section{Results}

\section{Immunohistochemistry}

Activation of Stat-3, Akt and Erk1/2 is accompanied by phosphorylation and can be detected using phospho-specific antibodies. ${ }^{22}$ Recently, antibodies designed to detect these phosphoproteins are commercially available and widely employed to evaluate their activity. In order to examine the localization and topographical relationship between EGFR expression and downstream molecules, IHC was performed for each protein. Results are shown in Figures 1 and 2 and Tables 1 and 2.

\section{Epidermal growth factor receptor}

EGFR staining was previously performed on 181 cases of NSCLC and 28 cases among them which were accompanied by fresh surgical tissues were reevaluated in this study. ${ }^{5}$ Seven cases $(25 \%)$ showed $2+$ staining confined to the cell membrane in more than $70 \%$ of the areas examined (Figure 1a and $b$ for Case 3), and five of them were known to harbor EGFR amplification (Figure 1d). ${ }^{5}$ Six cases $(21.4 \%)$ showed $1+$ staining both in the cell membrane as well as cytoplasm in $20-60 \%$ of the areas. The remaining 15 cases showed negative results (Figure $2 \mathrm{a}$ and $\mathrm{b}$ for Case 8, Tables 1 and 2).

\section{p-Epidermal growth factor receptor}

p-EGFR staining was very weakly $(1+)$ observed in five cases of tumors that showed $2+$ or $1+$ staining for EGFR (Table 2). However, positively stained areas were markedly localized, covering up to $5 \%$ of the areas.

\section{p-Signal transducers and activators of transcription-3}

In normal tissues, p-Stat-3 staining was occasionally observed in the nuclei of the basal cells in bronchial mucosa, alveolar epithelial cells and endothelial cells. In tumor tissue, p-Stat-3 was observed in the nuclei of cells located in the peripheral areas or invasive front of the tumor nest. Positive staining was observed in 12 cases, including seven cases of AC, four cases of SCC and one case of LC (Figure 1c for Case 3, Figure 2c for Case 8, Table 2).

\section{$p-A k t$}

In normal tissues, p-Akt staining was observed in the cytoplasm and nuclei of the bronchial epithelial cells. In tumor tissue, p-Akt staining was observed in both the cytoplasm and nuclei in 12 cases (42.9\%) (Table 2). Although cytoplasmic staining could be generally observed throughout the tumor nodule without any particular distribution pattern, nuclear staining was observed predominantly in the peripheral areas of the tumor nodule (Figure $2 \mathrm{~d}$ for Case 8).

p-Extracellular signal-related protein kinase 1/2 Although p-Erk1/2 staining was observed in both nuclei and the cytoplasm, nuclear staining was weakly observed in focal areas in five cases $(17.9 \%$, Table 2). The particular distribution pattern of the positively stained cells could not be evaluated.

In cases showing positive staining for any of these proteins, positively stained areas partially, but not completely overlapped with the EGFR-positive areas (Figures 1 and 2).

\section{Immunoblotting Analysis}

Next, expressions of EGFR as well as phosphoproteins were examined by IB to elucidate the potential role of these proteins in EGFR-mediated signaling. Results with densitometric analysis of the representative cases are presented in Figure 3, and the data of all the cases were shown in Tables 2 and 3.

\section{Epidermal growth factor receptor}

'Upregulation' of EGFR ('EGFR index'>1.5) was observed in 17 of 28 cases (60.7\%). Seven of these 17 cases showed strongly positive $(2+)$ staining for EGFR by IHC and five of these were previously confirmed to have EGFR amplification (Tables 1 and 2). ${ }^{5}$ Other six cases among these 17 showed weakly positive staining $(1+)$ and the remaining four cases showed negative staining by IHC. In adjacent normal tissues, 23 cases showed a low level of EGFR expression, in spite that no positive staining was observed in non-neoplastic tissues by IHC.

\section{p-Epidermal growth factor receptor}

'Activation' of EGFR was evaluated by blotting for EGFR phosphorylated at tyrosine ${ }^{1068}$, commonly used as a marker of EGFR autophosphorylation. ${ }^{10}$ By this assay, EGFR was found to be activated in 12 cases $(42.9 \%)$ all of which showed EGFR upregulation by IB. This leaves five cases that showed upregulation of total EGFR, yet did not show activation of EGFR. In normal tissues, 13 cases 

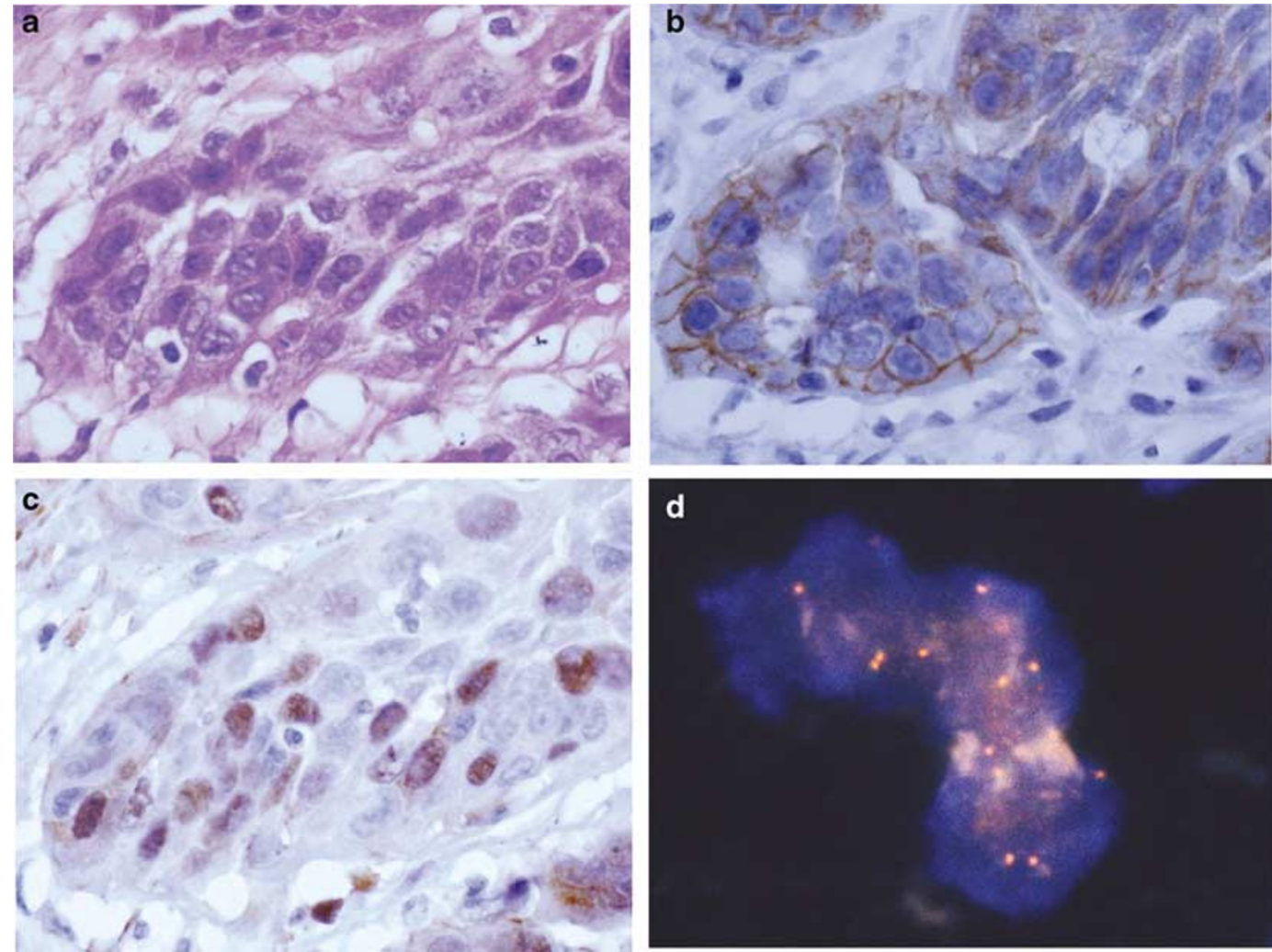

Figure 1 The results of immunohistochemical staining for EGFR and p-Stat-3 as well as FISH analysis. A case of squamous cell carcinoma (a, Hematoxylin-Eosin staining) which was confirmed to have EGFR amplification (Case 3), showing intense positive membrane staining of EGFR (b), and positive nuclear staining for p-Stat-3 (c) in the peripheral area of the tumor nest. (d) Tumor cells with four copies of scattered signals of EGFR (orange) were observed by FISH analysis. Original magnification, $\times 400$.
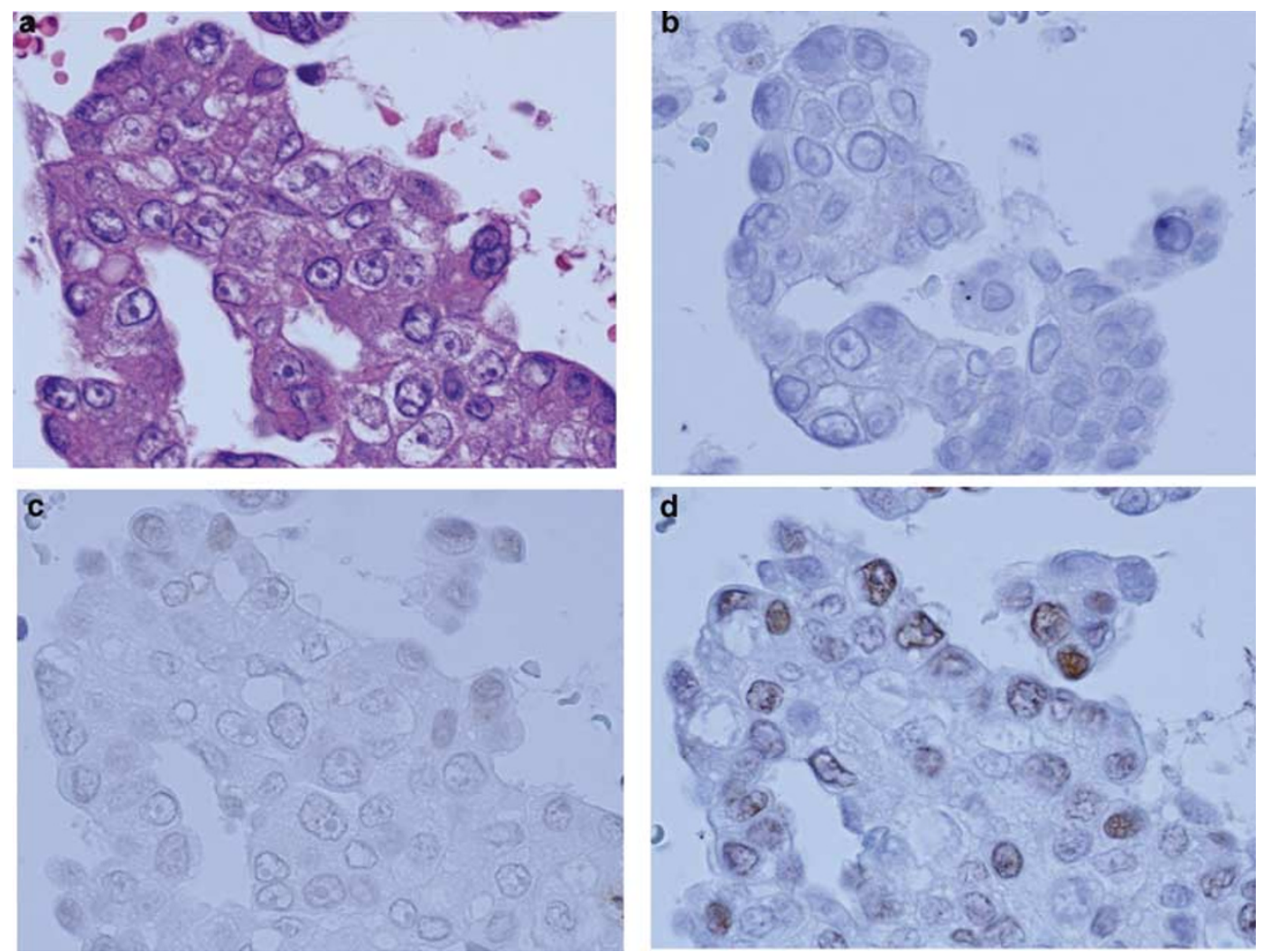
Table 2 Overall data of the tissues examined

\begin{tabular}{|c|c|c|c|c|c|c|c|c|c|c|c|c|c|c|c|}
\hline \multirow[t]{2}{*}{ Case } & \multicolumn{2}{|c|}{ Histology } & \multicolumn{5}{|c|}{ Immunohistochemistry } & \multicolumn{5}{|c|}{ Immunobltting analysis } & \multirow[t]{2}{*}{$F I S H^{\mathrm{a}}$} & \multirow[t]{2}{*}{ Mutation } & \multirow[t]{2}{*}{ Metastasis } \\
\hline & & & $E G F R$ & $p-E G F R$ & $p$-Stat3 & $p-A k t$ & p-Erk & $E G F R$ & $p-E G F R$ & $p-S t a t 3$ & $p-A k t$ & $p$-Erk & & & \\
\hline 1 & $\mathrm{AC}^{\mathrm{b}}$ & $\operatorname{Mix}^{\mathrm{c}}$ & $2+$ & $1+$ & $1+$ & - & - & $7.33^{\mathrm{d}}$ & 4.00 & 3.15 & 1.25 & 0.44 & + & & + \\
\hline 2 & $\mathrm{SCC}^{\mathrm{e}}$ & & $2+$ & - & $1+$ & - & - & 4.86 & 1.33 & 4.67 & 1.38 & 2.24 & + & & + \\
\hline 3 & SCC & & $2+$ & - & $1+$ & - & - & 6.25 & 1.85 & 3.15 & 1.00 & 0.55 & + & & \\
\hline 4 & SCC & & $2+$ & - & $1+$ & - & - & 7.00 & 1.90 & 2.93 & 1.14 & 1.00 & + & & \\
\hline 5 & $\mathrm{AC}$ & Mix & $2+$ & $1+$ & $1+$ & - & - & 5.60 & 3.50 & 3.64 & 1.00 & 0.59 & + & S695R & \\
\hline 6 & $\mathrm{AC}$ & Mix & - & - & - & $1+$ & - & 1.63 & 5.00 & 1.25 & 2.32 & 2.09 & & del747-753insS & + \\
\hline 7 & $\mathrm{AC}$ & Mix & $1+$ & $1+$ & - & $1+$ & - & 2.40 & 4.10 & 1.38 & 4.90 & 1.00 & & del747-752 & + \\
\hline 8 & $\mathrm{AC}$ & Mix & - & - & - & $1+$ & - & 2.20 & 2.75 & 1.09 & 4.13 & 1.06 & & L858R & + \\
\hline 9 & $\mathrm{AC}$ & Mix & $1+$ & $1+$ & - & $1+$ & - & 3.29 & 5.00 & 1.13 & 3.33 & 0.65 & & L858R & \\
\hline 10 & SCC & & $2+$ & - & $1+$ & - & - & 2.80 & 1.90 & 3.80 & 1.18 & 1.20 & & & \\
\hline 11 & SCC & & $2+$ & - & - & - & $1+$ & 4.29 & 1.57 & 1.08 & 1.04 & 2.23 & & & \\
\hline 12 & $\mathrm{AC}$ & Mix & $1+$ & - & $1+$ & - & - & 2.25 & 1.30 & 2.60 & 1.25 & 2.20 & & & \\
\hline 13 & $\mathrm{AC}$ & Mix & $1+$ & - & $1+$ & - & - & 1.90 & 1.90 & 2.40 & 0 & 1.60 & & & \\
\hline 14 & $\mathrm{AC}$ & Mix & $1+$ & - & - & - & - & 1.60 & 1.25 & 2.86 & 1.31 & 0.50 & & & \\
\hline 15 & SCC & & $1+$ & - & - & $1+$ & - & 2.60 & 0 & 1.17 & 1.67 & 0.93 & & & + \\
\hline 16 & $\mathrm{AC}$ & Mix & - & - & $1+$ & $1+$ & - & 0 & 1.25 & 3.33 & 1.70 & 0.50 & & & \\
\hline 17 & $\mathrm{AC}$ & Mix & - & - & - & - & - & 0 & 0 & 1.29 & 1.60 & 1.50 & & & \\
\hline 18 & $\mathrm{AC}$ & Mix & - & - & - & - & - & 1.70 & 2.00 & 2.00 & 1.29 & 1.10 & & & \\
\hline 19 & $\mathrm{AC}$ & Mix & - & - & - & $1+$ & - & 1.40 & 0 & 1.40 & 3.60 & 0.50 & & & + \\
\hline 20 & $\mathrm{AC}$ & Mix & - & - & - & - & $1+$ & 1.33 & 1.22 & 1.11 & 1.60 & 2.70 & & & \\
\hline 21 & $\mathrm{AC}$ & Mix & - & - & $1+$ & - & - & 1.20 & 0 & 4.25 & 1.22 & 0.50 & & & \\
\hline 22 & $\mathrm{AC}$ & $\operatorname{Pap}^{f}$ & - & - & $1+$ & - & $1+$ & 1.25 & 0 & 2.30 & 1.20 & 2.00 & & & \\
\hline 23 & $\mathrm{AC}$ & Solidg & - & - & - & - & - & 1.43 & 1.10 & 1.25 & 1.00 & 2.50 & & & \\
\hline 24 & SCC & & - & - & - & $1+$ & $1+$ & 1.25 & 1.00 & 1.10 & 1.60 & 4.30 & & & \\
\hline 25 & SCC & & - & - & - & $1+$ & - & 1.29 & 1.43 & 1.00 & 2.00 & 1.50 & & & \\
\hline 26 & SCC & & - & - & - & $1+$ & - & 1.63 & 0 & 1.22 & 2.00 & 1.50 & & & \\
\hline 27 & SCC & & - & - & - & $1+$ & $1+$ & 1.11 & 0 & 1.30 & 2.90 & 3.10 & & & + \\
\hline 28 & $\mathrm{LC}^{\mathrm{h}}$ & & - & - & $1+$ & $1+$ & - & 1.00 & 0 & 1.82 & 2.13 & 1.25 & & & \\
\hline Cont1 & bulla & & - & - & - & - & - & 0.30 & 0 & 0.29 & 0.11 & 0.90 & & & \\
\hline Cont2 & bulla & & - & - & - & - & - & 0.20 & 0 & 0.19 & 0.15 & 1.20 & & & \\
\hline
\end{tabular}

${ }^{\mathrm{a}} \mathrm{FISH}$, presence of amplification by fluorescein in situ hybridization analysis.

${ }^{\mathrm{b}} \mathrm{AC}$, adenocarcinoma.

${ }^{\mathrm{C}} \mathrm{Mix}$, adenocarcinoma of mixed subtype.

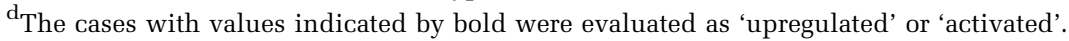

${ }^{\text {e }}$ SCC, squamous cell carcinoma.

${ }^{\mathrm{f}}$ Pap, papillary adenocarcinoma.

gSolid, solid adenocracinoma with mucin.

$\mathrm{h}_{\mathrm{LC}}$, large cell carcinoma.

showed detectable p-EGFR, but bullous lung tissue did not show any detectable p-EGFR signal.

\section{p-Signal transducers and activators of transcription-3}

Activation of Stat-3 was observed in 14 cases and seven of these 14 cases showed EGFR activation. Thus, the remaining seven cases showed activation of Stat-3 without accompanying activation of EGFR, presumably by signals through a cascade that does not involve EGFR.

In normal tissues, 25 cases showed detectable p-Stat-3, whereas non-neoplastic tissues from cancer patients showed slightly higher values compared with the bullous lung tissue $(0.80$ vs 0.24 in average).

\section{$p-A k t$}

Activation of Akt was observed in 14 cases (50\%). Four of these 14 cases also showed activation of EGFR. Tumor tissues and adjacent normal tissues revealed average p-Akt values of 2.42 and 1.07, respectively, but bullous lung tissue showed a very low level of p-Akt values of 0.11 and 0.15 .

p-Extracellular signal-related protein kinase 1/2 Activation of Erk1/2 was observed in 13 of 28 cases of tumors $(46.4 \%)$. Only three cases of these 13 cases

Figure 2 The results of immunohistochemical staining for EGFR, p-Stat-3 and p-Akt. A case of adenocarcinoma (a, hematoxylin-eosin staining) which has an EGFR point mutation, but not amplification (Case 8). IHC against EGFR protein (b), p-Stat-3 (c) and p-Akt (d), showing intense positive nuclear staining for p-Akt in the invasive front of the tumor. However, detectable level of staining for EGFR or p-Stat-3 was not observed. Original magnification, $\times 400$. 


\begin{tabular}{|c|c|c|c|c|c|c|c|c|c|c|c|c|c|c|c|c|c|c|c|c|c|c|c|c|c|}
\hline Case No. & $1 *$ & & & $2 *$ & & 3 & & 4 & & 5 & & * & & * & & $*$ & 9 & & & 1 & & 5* & \multicolumn{3}{|c|}{28} \\
\hline \multirow{5}{*}{$\begin{array}{c}\text { EGFR-IHC } \\
\text { Amplification } \\
\text { Mutation }\end{array}$} & $2+$ & & & $2+$ & 2 & + & & $2+$ & & $2+$ & & - & 1 & + & . & - & $1+$ & & 2 & $2+$ & 1. & + & - & & - \\
\hline & + & & & + & & + & & + & & + & & - & - & & 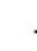 & - & - & & . & - & & - & - & & - \\
\hline & - & & & - & & - & & - & & + & & + & + & & . & + & + & & . & - & . & - & - & & - \\
\hline & A & C & SC & & $\mathrm{sc}$ & & SC & $\mathrm{CC}$ & & $A C$ & & $A C$ & & C & & $A C$ & $\mathrm{AC}$ & C & $\mathrm{SC}$ & $\mathrm{CC}$ & & $\mathrm{CC}$ & $\mathrm{LC}$ & $C$ & bulla \\
\hline & $\mathrm{N}$ & $\mathrm{T}$ & $\mathrm{N}$ & $\mathrm{T}$ & $\mathrm{N}$ & $T$ & $N$ & $T$ & $\mathrm{~N}$ & $T$ & $N$ & $T$ & $\mathrm{~N}$ & $\mathrm{~T}$ & $\mathrm{~N}$ & $T$ & $\mathrm{~N}$ & $\mathrm{~T}$ & $\mathrm{~N}$ & $\mathrm{~T}$ & $N$ & $T$ & $\mathrm{~N}$ & $\mathrm{~T}$ & C \\
\hline EGFR & & - & - & - & - & - & - & - & & - & - & - & & - & & - & - & - & - & - & 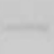 & - & - & & - \\
\hline & 0.3 & 2.2 & 0.7 & 3.4 & 0.8 & 5.0 & 0.6 & 4.2 & 0 & 5.6 & 0.8 & 1.3 & $c$ & 2.4 & 0 & 2.2 & 0.7 & 2.3 & 0.7 & 3.0 & 0.5 & 1.3 & 0.6 & 0.6 & 0.6 \\
\hline
\end{tabular}
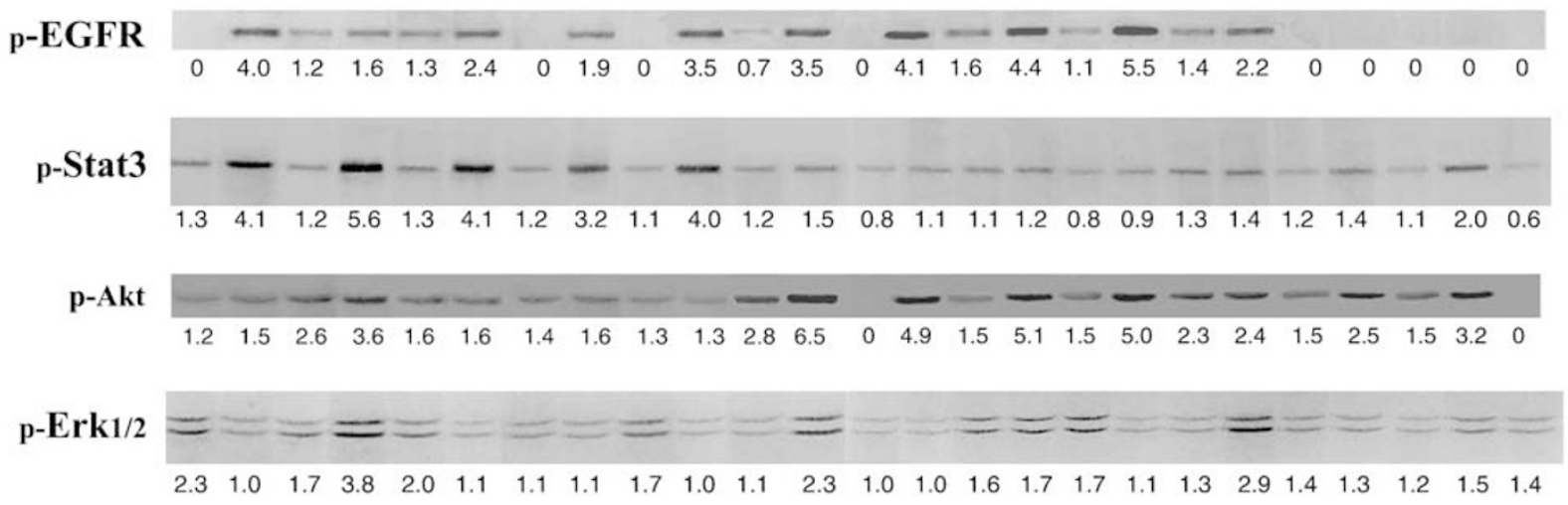

$\beta$-actin

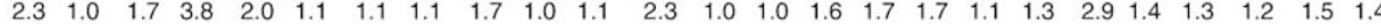

Figure 3 Protein levels of EGFR, p-EGFR, pStat-3, p-Akt and p-Erk1/2 evaluated by immunoblotting analysis in representative cases of tumor and paired normal tissue. The intensity of the band was quantitated and expressed as ratio relative to that of $\beta$-actin, designated as 10. In the case of Erk1/2, the fastest migrating bands were subjected to quantification. Asterisks indicate the cases showing lymph node metastasis and ' $\mathrm{C}$ ' in the lane of bulla represents 'control'.

Table 3 Results of immunoblotting analysis (cases)

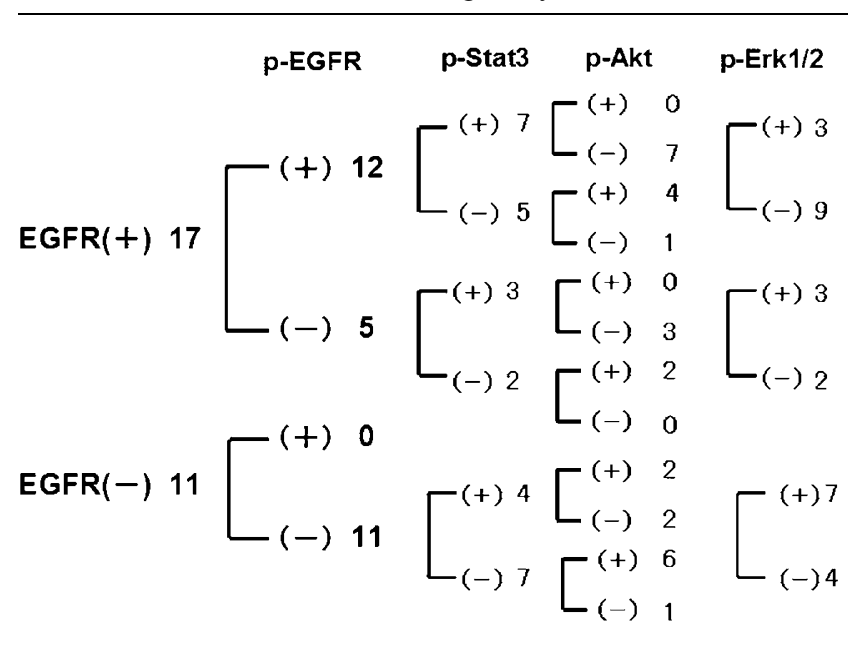

showed accompanying activation of EGFR. Thus, the remaining 10 cases were p-EGFR negative, suggesting that phosphorylation of Erk1/2 could be inversely related to EGFR activation. In 12 cases of the remaining 15 cases that did not show Erk1/2 activation in tumors, adjacent normal tissue re- vealed identical or even higher levels of $p$-Erk1/2 than in the tumor tissue. Tissues from the bullous lung tissue also showed Erk1/2 phosphorylation at levels equivalent to those of normal tissue from cancer patients.

\section{EGFR Mutation Spectrum}

Given the apparent clustering of EGFR mutations as described previously, ${ }^{10,11}$ we sequenced exons 18-21 in 28 primary tumors. Sequencing of PCR-amplified DNA fragments revealed mutations in five cases. Genes from two tumors contained in-frame deletions within exon 19, removing amino acids 747 to 753 (delL747-P753insS) in Case 6 and 747 to 752 (delL747-S752) in Case 7 (Figure 4a). The former resulted in the insertion of a serine, owing to the generation of a novel codon at the deletion breakpoint. Three other tumors had amino-acid substitutions: the substitution of $\mathrm{T}$ for $\mathrm{G}$ at nucleotide 2085 resulted in substitution of serine to arginine at codon 695 (S695R) of exon 18 in Case 5 (Figure 4b), and leucine to arginine at codon 858 (L858R) of exon 21 in cases 8 and 9 (Figure 4c). Mutations were found once only in each case and 
the paired normal lung tissue showed wild-type sequence, confirming that the mutations are somatic in origin.

All of the above mutations were found in the cases of 'adenocarcinoma of the mixed subtype'. The frequency was $17.9 \%(5 / 28)$ in overall NSCLC and $29.4 \%(5 / 17)$ in AC.

\section{Correlation among the Results of Gene Aberrations, Protein Expression and Phosphorylation of Downstream Molecules}

The overall results obtained from these different analyses were combined for all of the cases. EGFR amplification was found in five cases (Cases 1-5). These cases exhibited higher levels of EGFR expression (4.86-7.33 as indices) and four of these cases showed activation of EGFR (1.85-4.0). Therefore, in the tumors exhibiting EGFR gene amplification, EGFR protein expression was markedly higher, and often activated. In all these five cases, Stat-3 was activated (2.67-4.67 of p-Stat-3 index) to significantly higher level than the remaining 23 cases $(P<0.005)$, but Erk1/2 were activated only in one case and Akt was never activated. One case harboring amplification as well as mutation (Case 5), Stat-3 was activated, but Akt was not despite the presence of mutation.

Mutation was found in five cases (Cases 5-9) one of which showed gene amplification (Case 5). This indicates that the mutation could be, but not necessarily associated with amplification. Although this particular case showed remarkably high expression of EGFR (5.60 of EGFR-index), probably due to amplification, four other tumors showed slightly higher expression of EGFR compared to other tumors (1.63-3.29 of EGFR-index), but not as high as the tumors showing amplification. However, these four tumors with mutated EGFR, but no amplification, showed markedly increased level of activated EGFR, with the p-EGFR indices ranging from 2.75 to 5.0. p-Akt was detectable at significantly higher levels (2.32-4.9 of p-Akt index, $P<0.001)$ compared with the remaining 24 cases, and was thus considered to be activated in those four cases, whereas Stat-3 was never activated and Erk1/2 was activated in only one case (Case 6). Therefore, mutation of EGFR not accompanied by gene amplification was associated with slightly higher EGFR expression and significantly higher activation state of both EGFR itself and Akt.

In the remaining 19 cases, EGFR expression was upregulated in eight cases $(42.1 \%)$, but the levels were far lower (2.35 of an average index) than those cases exhibiting gene amplification (6.21 of an average index). In those eight cases, a specific correlation with activation of downstream molecules was not observed. Overall, Stat-3, Akt and Erk1/2 were activated in 14, 14 and 13 cases, respectively. Each case showed activation of at least one of these three phosphoproteins. In particular, the phosphorylation patterns of Stat-3 and Akt were reciprocal and complementary: 24 cases showed activation of either of Stat-3 or Akt, and only two cases showed both.

\section{Clinicopathological Analysis}

These experimental results were statistically evaluated with clinical profiles of the cases. First, with regards to metastasis, correlation between IHC expression of each protein and metastasis was not found. However, when metastatic potential was analyzed in correlation with protein expression levels evaluated by IB, there was the trend that the cases showing lymph node metastasis revealed higher p-Akt expression (Table 2). Among eight cases showing lymph node metastasis, all cases revealed p-Akt indices of higher than 1.25 with the average of 2.68 and six of those eight cases were interpreted as 'activated'. In those eight cases, EGFR was activated in four cases, Stat-3 in two cases and Erk1/2 in three cases. In contrast, among 20 cases without metastasis, cases showing activated Akt were observable only in eight cases, and the average index was 2.00. Even in this limited sample size, the difference of Akt phosphorylation between these two groups was statistically significant $(P<0.05)$. Thus, Akt phosphorylation was a possible predictive factor of metastasis. However, mutation of EGFR, expression levels of EGFR, phosphorylation of EGFR, Stat-3 or Erk1/2 did not show correlation with metastasis.

Next, IHC/IB expression of each protein was evaluated for the potential prognostic significance. Univariate analysis revealed no significant difference in survival rates between IHC-positive and negative groups or high- and low index groups evaluated by IB in any of proteins, probably due to small sample size.

\section{Discussion}

Gefitinib has been in clinical use in Japan and the United States for the treatment of NSCLC for the past several years. ${ }^{3,8}$ However, EGFR overexpression per se reflects the less critical role in determining the responsiveness to gefitinib. ${ }^{13,14,36}$ In fact, the relationship between the presence of amplification and responsiveness to gefitinib as well as overall survival has been still under controversy depending on the reports. ${ }^{13,14,36}$ It is, nowadays, consensus that the mutation in EGFR predominantly determines the effectiveness. This means that in only a subgroup of cancers which harbor EGFR mutations, EGFR signaling may play a critical role in biological behavior. Although experiments using cell lines harboring amplified or mutant EGFR or transfection of EGFR on cultured cells will show us precise underlying 

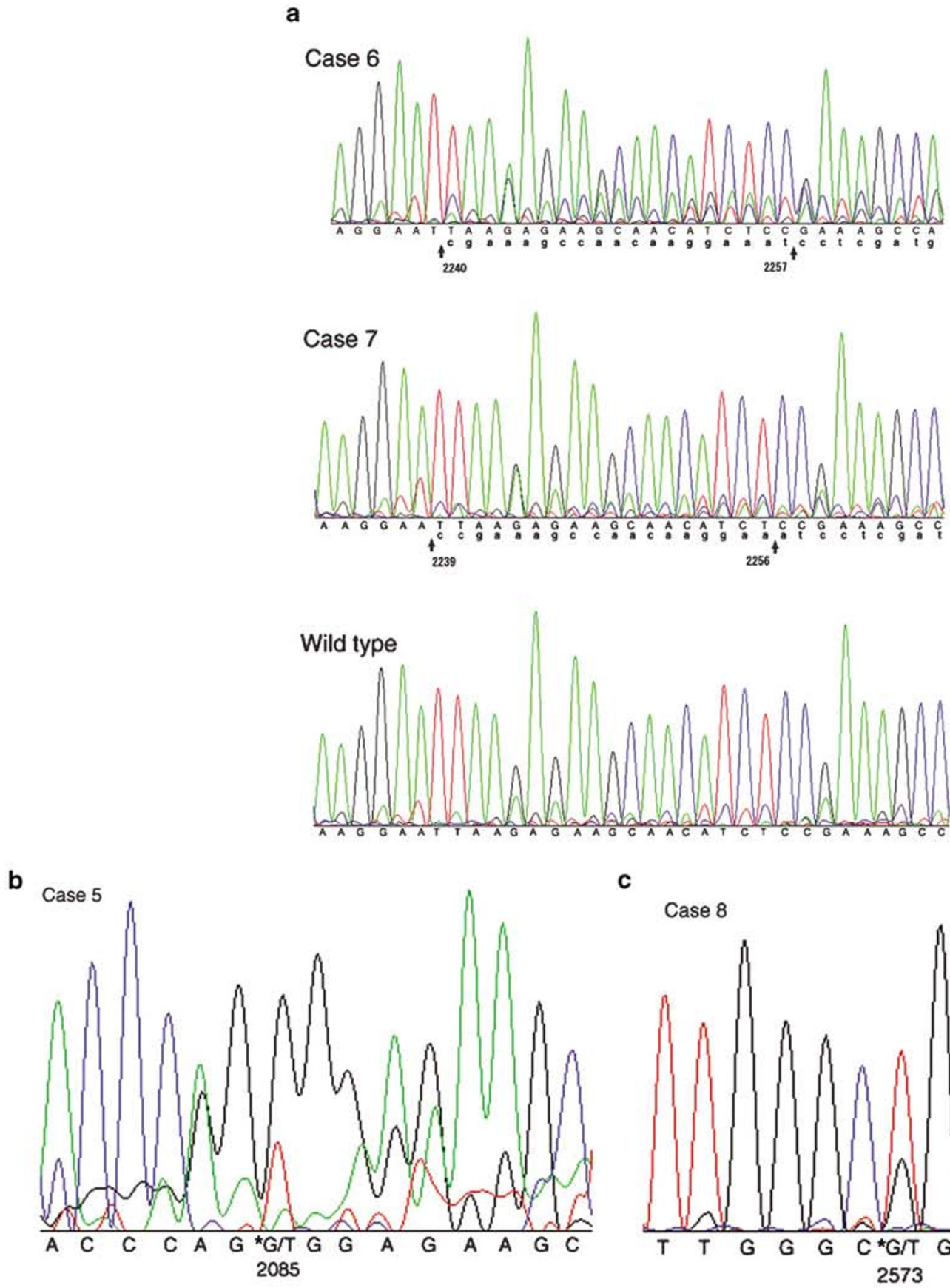

c
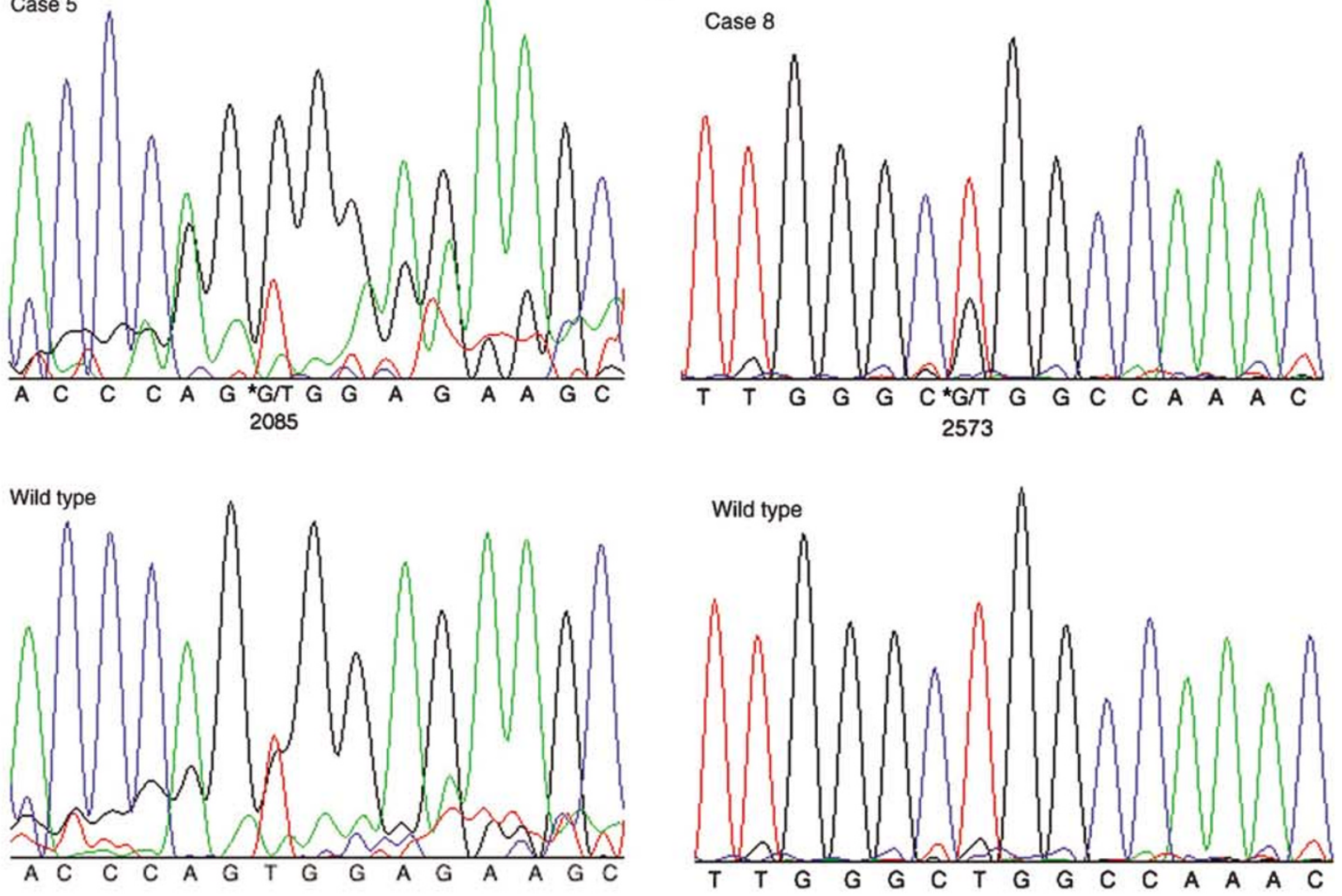
mechanisms of signal transduction, those models may not necessarily embody signaling events in real human tumors. Thus, we performed this study using many cases of human lung carcinomas.

Accumulated evidences have already shown that three downstream molecules of EGFR are actively involved in survival, proliferation and/or progression of tumor cells. ${ }^{22}$

Close relationship between activation of Stat-3 and EGFR has been described in many human tumors, ${ }^{37,38}$ although EGFR-independent activation of Stat-3 in vivo and in vitro has also been described. ${ }^{21,22}$ Moreover, a positive correlation has been observed between EGFR mRNA or protein levels and Stat-3 activation in cultured cells. ${ }^{37}$ Based on the present results showing that Stat-3 phosphorylation could be detected in cases exhibiting amplification of EGFR, strategies that selectively abrogate Stat-3 could be considered as targeting therapy against the tumors exhibiting EGFR amplification.

The PI3K-Akt pathway is partially involved in promoting cell motility and invasiveness of tumor cells, and is associated with metastasis in human tumors. ${ }^{39}$ Indeed, we found that the cases exhibiting lymph node metastasis generally had higher p-Akt levels. However, Akt activation has been variously reported to be associated with better prognosis, ${ }^{28}$ or with a better response to gefitinib, ${ }^{13}$ but there have also been other reports indicating that Akt expression is correlated to resistance against chemotherapy and radiation. ${ }^{26,36}$ In turn, Akt activation was also shown to be closely associated with mutation of EGFR. In a report using cultured cells transfected with mutant EGFR, the PI3K-Akt and Stat pathways, but not the Erk1/2 pathway, were found to be activated by EGF stimulation. ${ }^{12}$ Consistently, our results using surgical specimens of human NSCLC revealed that mutation of EGFR is closely associated with activation of Akt.

Stat-3, Akt and Erk1/2 each plays a particular role in promoting and maintaining tumors via a complicated network of downstream molecules involving crosstalk among several signaling cascades. ${ }^{3,6,12,13}$ The overall results in this study suggest that Stat-3 and Akt were activated in a reciprocal and complementary manner in both human NSCLC: that is, in 24 of 28 cases, either of Stat-3 or Akt, but not both, was activated. In contrast, Erk1/2 was activated regardless of the status of Stat-3 or Akt, and did not show any specific correlations with the status of upstream molecules, such as EGFR mutation, amplification or overexpression of EGFR protein.

One explanation for the diversity of molecules that are predominantly activated is that established cancer cells must maintain homeostasis between positive- and negative-acting factors, and thus the particular signaling cascade that is predominantly activated differs depending on the stage of the tumor and its environment. Furthermore, the cell apparently has fail-safe mechanisms against aberrant stimuli by growth factor, constitutive activation of downstream molecules and subsequent transformation. For example, the C-tail of EGFR plays an important role in preventing constitutive Stat activation, and following stimulation, EGFR as well as downstream molecules seem to be immediately downregulated by dephosphorylation and/or internalization. ${ }^{3,40}$ Collectively, the levels, duration and other parameters of various mitogenic signals seem to determine whether cells undergo proliferation or senescence/apoptosis in response to EGFR signals mediated by Stat-3, Akt and Erk1/2. Under these complicated environments, our findings showing Stat-3 activation in EGFR-amplified tumors, and Akt activation in mutant EGFR harboring tumors, may be notable in that they strongly indicate the specific activation of those two molecules in particular subsets of NSCLC. In these groups of carcinomas, abnormality of EGFR may cause sustained activation of Stat-3 or Akt even though half-life of phosphoproteins are usually short..$^{3,40}$

There was one tumor that exhibited both EGFR amplification and mutation (Case 5). In this case, Stat-3, rather than Akt was activated despite the presence of mutations. This may be because the signal from amplified and overexpressed EGFR through Stat-3 was dominant. With respect to sensitivity to gefitinib, mutants harboring deletions in exon 19 (cases 6 and 7) or L858R in exon 21 (cases 8 and 9) have been shown to predict responsiveness, ${ }^{7,10,11}$ although the susceptibility of tumors containing mutation in S695R (Case 5) has not been described previously. One hypothesis is that mutants sensitive for gefitinib transduce signals mediated by Akt and that the mutants resistant for gefitinib do not. If this is true, the tumor in Case 5 may not show sensitivity to gefitinib. However, clarification of this hypothesis requires further study.

p-Erk1/2 expression was not correlated with activation of EGFR in NSCLC, as also observed

Figure 4 Mutations in the EGFR gene. (a) Nucleotide sequence of the EGFR gene in tumor specimens with in-frame deletions within exon 19. Both Case 6 (upper row) and Case 7 (middle row) are shown to indicate the breakpoints of the deletion by arrows; the wild-type nucleotide sequence is shown in uppercase letters, and the mutant sequence is in lowercase letters. Only the sequence of the same region from the paired normal tissue from Case 6 is presented at the bottom. The $5^{\prime}$ breakpoint of the delL747-P753insS mutation in the upper row is preceded by a residual T followed by CG substitution for TA, resulting in a novel codon insertion encoding serine. (b) and (c) Missense mutations (asterisk) resulting in amino-acid substitutions from serine to arginine at codon 695 in Case 5 (b) and leucine to arginine at codon 858 in Case 8 (c). The double peaks represent two nucleotides at the site of heterozygous mutations. The corresponding wild-type sequences from matched normal tissue are shown below. 
in our previous study on bone and soft tissue tumors. ${ }^{30}$

In adjacent normal tissue from cancer-bearing lung, Erk1/2 was frequently phosphorylated (25 cases), followed by Stat-3 and Akt. In addition, those normal tissues occasionally showed similar or higher levels of p-Erk1/2 compared with the tumor tissues. Consistently, histologically normal mucosa from the patients, unlike normal epithelium from patients without cancer, have been reported to show upregulation of EGFR signaling proteins. ${ }^{23,29}$ This may be because adjacent normal tissue is activated by growth factors from the tumor in a paracrine manner, or these cells could have a metabolically higher condition owing to a higher blood supply and immunological reaction against the tumor, as none of phosphoproteins was highly phosphorylated in the tissue from bullous lung.

Although mutation of EGFR is generally associated with susceptibility to gefitinib, even these responsive tumors are not completely eradicated by gefitinib. Therefore, these cancers could potentially be treated by initially targeting the putative crucial molecule, such as mutated EGFR as mutated proteins may constitute the Achilles' heels in human cancer treatment. ${ }^{3}$ However, in the subsequent treatment, downstream activated molecules should next be targeted as they may be more critical for survival. Although this study was on a small scale, the results showed clear trends and suggest several implications for therapy. First, amplification and mutation could be, but not necessarily associated with each other, as one of the five cases with mutation of EGFR showed gene amplification. This is comparable to data from a previous report showing that approximately $15-40 \%$ of tumors with mutations have amplification of the mutant allele. ${ }^{3,18,41}$ Second, tumors with amplification of the EGFR gene expressed significantly higher amounts of EGFR protein compared with other cases, and EGFR was often activated. This enhanced EGFR signal is mediated by Stat-3, therefore, these cases could be indication for the treatment with Stat-3 inhibitors (tyrphostin AG17, cucurbitacin, etc), in addition to cetuximab which may contribute to efficacy as it may inhibit the action of overexpressed EGFR protein. ${ }^{42}$ Third, tumors having mutated EGFR did not show remarkable overexpression of EGFR compared with tumors harboring EGFR amplification, yet EGFR was highly phosphorylated and signaled predominantly through Akt. It has been consistently reported that sensitivity to growth inhibition by gefitinib in NSCLC cell lines is associated with dependence on Akt and Erk1/2 activation. ${ }^{15}$ Therefore, tumors with mutation of EGFR could be treated with Gefinitib, and in addition with Akt antagonist and PI3K inhibitors (KP372, LY294002 and wortmannin), which may enhance inhibition of the signaling cascade. ${ }^{43,44}$ In the rest of the cases, the downstream molecules that are specifically and selectively activated should be identified. Precise screening for these phenotypes could identify which patients may have a good response to targeting therapy and eventually determine the appropriate therapeutic strategy for each patient.

As the present study examined surgically resected cases that had not been subjected to gefitinib therapy, the correlation between our results and clinical responsiveness was not directly evaluated. Some important questions remain to be answered: (i) whether tumors harboring different EGFR mutants, and thus having different sensitivity against gefitinib still show activation of p-Akt, (ii) why the effect of mutation and amplification on downstream cascades was different and (iii) whether tumors showing constitutive activation of Stat-3 or Akt really respond to their cognate inhibitors. Analyses for answering these questions using mutated EGFR cDNA in cultured cells are required and have been under way in our laboratory.

\section{Acknowledgements}

This work was supported by the Japanese Ministry of Education, Sports, Science and Culture no. C 16590272, C 17590298, C 18590327 and the research grant from the Yamanashi Academy of Science.

\section{References}

1 Jemal A, Thomas A, Murray T, et al. Cancer statistics, 2002. CA Cancer J Clin 2002;52:23-47.

2 Bunn Jr PA, Franklin W. Epidermal growth factor receptor expression, signal pathway, and inhibitors in non-small cell lung cancer. Semin Oncol 2002;29: 38-44.

3 Pao W, Miller VA. Epidermal growth factor receptor mutations, small-molecule kinase inhibitors, and nonsmall-cell lung cancer: current knowledge and future directions. J Clin Oncol 2005;23:2556-2568.

4 Pegram MD, Konecny G, Slamon DJ. The molecular and cellular biology of HER2/neu gene amplification/ overexpression and the clinical development of herceptin (trastuzumab) therapy for breast cancer. Cancer Treat Res 2000;103:57-75.

5 Suzuki S, Dobashi Y, Sakurai H, et al. Protein overexpression and gene amplification of epidermal growth factor receptor in nonsmall cell lung carcinomas. An immunohistochemical and fluorescence in situ hybridization study. Cancer 2005;103:1265-1273.

6 Arteaga CL. Overview of epidermal growth factor receptor biology and its role as a therapeutic target in human neoplasia. Semin Oncol 2002;29:3-9.

7 Pao W, Miller V, Zakowski M, et al. EGF receptor gene mutations are common in lung cancers from 'never smokers' and are associated with sensitivity of tumors to gefitinib and erlotinib. Proc Natl Acad Sci USA 2004;101:13306-13311.

8 Fukuoka M, Yano S, Giaccone G, et al. Multi-institutional randomized phase II trial of gefitinib for 
previously treated patients with advanced non-smallcell lung cancer (The IDEAL 1 Trial) [corrected]. J Clin Oncol 2003;21:2237-2246.

9 Huang SF, Liu HP, Li LH, et al. High frequency of epidermal growth factor receptor mutations with complex patterns in non-small cell lung cancers related to gefitinib responsiveness in Taiwan. Clin Cancer Res 2004;10:8195-8203.

10 Lynch TJ, Bell DW, Sordella R, et al. Activating mutations in the epidermal growth factor receptor underlying responsiveness of non-small-cell lung cancer to gefitinib. N Engl J Med 2004;350:2129-2139.

11 Paez JG, Janne PA, Lee JC, et al. EGFR mutations in lung cancer: correlation with clinical response to gefitinib therapy. Science 2004;304:1497-1500.

12 Sordella R, Bell DW, Haber DA, et al. Gefitinibsensitizing EGFR mutations in lung cancer activate anti-apoptotic pathways. Science 2004;305: 1163-1167.

13 Cappuzzo F, Magrini E, Ceresoli GL, et al. Akt phosphorylation and gefitinib efficacy in patients with advanced non-small-cell lung cancer. J Natl Cancer Inst 2004;96:1133-1141.

14 Baselga J, Rischin D, Ranson M, et al. Phase I safety, pharmacokinetic, and pharmacodynamic trial of ZD1839, a selective oral epidermal growth factor receptor tyrosine kinase inhibitor, in patients with five selected solid tumor types. J Clin Oncol 2002; 20:4292-4302.

15 Ono M, Hirata A, Kometani T, et al. Sensitivity to gefitinib (Iressa, ZD1839) in non-small cell lung cancer cell lines correlates with dependence on the epidermal growth factor (EGF) receptor/extracellular signal-regulated kinase $1 / 2$ and EGF receptor/Akt pathway for proliferation. Mol Cancer Ther 2004;3:465-472.

16 Tsao MS, Sakurada A, Cutz JC, et al. Erlotinib in lung cancer-molecular and clinical predictors of outcome. N Engl J Med 2005;353:133-144.

17 Hirsch FR, Varella-Garcia M, McCoy J, et al. Increased epidermal growth factor receptor gene copy number detected by fluorescence in situ hybridization associates with increased sensitivity to gefitinib in patients with bronchioloalveolar carcinoma subtypes: a Southwest Oncology Group Study. J Clin Oncol 2005;23: 6838-6845.

18 Cappuzzo F, Hirsch FR, Rossi E, et al. Epidermal growth factor receptor gene and protein and gefitinib sensitivity in non-small-cell lung cancer. J Natl Cancer Inst 2005;97:643-655.

19 Cappuzzo F, Varella-Garcia M, Shigematsu H, et al. Increased HER2 gene copy number is associated with response to gefitinib therapy in epidermal growth factor receptor-positive non-small-cell lung cancer patients. J Clin Oncol 2005;23:5007-5018.

20 Lin AW, Barradas M, Stone JC, et al. Premature senescence involving p53 and p16 is activated in response to constitutive MEK/MAPK mitogenic signaling. Genes Dev 1998;12:3008-3019.

21 Levy DE, Darnell Jr JE. Stats: transcriptional control and biological impact. Nat Rev Mol Cell Biol 2002;3:651-662.

22 Bowman T, Garcia R, Turkson J, et al. STATs in oncogenesis. Oncogene 2000;19:2474-2488.

23 Grandis JR, Drenning SD, Zeng Q, et al. Constitutive activation of Stat3 signaling abrogates apoptosis in squamous cell carcinogenesis in vivo. Proc Natl Acad Sci USA 2000;97:4227-4232.
24 Garcia R, Jove R. Activation of STAT transcription factors in oncogenic tyrosine kinase signaling. J Biomed Sci 1998;5:79-85.

25 Song L, Turkson J, Karras JG, et al. Activation of Stat3 by receptor tyrosine kinases and cytokines regulates survival in human non-small cell carcinoma cells. Oncogene 2003;22:4150-4165.

26 Brognard J, Clark AS, Ni Y, et al. Akt/protein kinase B is constitutively active in non-small cell lung cancer cells and promotes cellular survival and resistance to chemotherapy and radiation. Cancer Res 2001;61: 3986-3997.

27 Sakoda H, Gotoh Y, Katagiri H, et al. Differing roles of Akt and serum- and glucocorticoid-regulated kinase in glucose metabolism, DNA synthesis, and oncogenic activity. J Biol Chem 2003;278:25802-25807.

28 Shah A, Swain WA, Richardson D, et al. Phospho-akt expression is associated with a favorable outcome in non-small cell lung cancer. Clin Cancer Res 2005;11:2930-2936.

29 Hoshino R, Chatani Y, Yamori T, et al. Constitutive activation of the $41-/ 43-\mathrm{kDa}$ mitogen-activated protein kinase signaling pathway in human tumors. Oncogene 1999;18:813-822.

30 Dobashi Y, Takei N, Suzuki S, et al. Aberration of epidermal growth factor receptor expression in bone and soft-tissue tumors: protein overexpression, gene amplification and activation of downstream molecules. Mod Pathol 2004;17:1497-1505.

31 Pao W, Miller VA, Politi KA, et al. Acquired resistance of lung adenocarcinomas to gefitinib or erlotinib is associated with a second mutation in the EGFR kinase domain. PLoS Med 2005;2:e73.

32 Kobayashi S, Boggon TJ, Dayaram T, et al. EGFR mutation and resistance of non-small-cell lung cancer to gefitinib. N Engl J Med 2005;352:786-792.

33 Travis WD, Colby TV, Corrin B, et al (eds). In collaboration with L.H. Sobin and Pathologists from 14 Countries. World Health Organization. Histological Typing of Lung and Pleural Tumors. International Histological Classification of Tumours. 3rd edn. Springer: New York, 1999.

34 Shia J, Klimstra DS, Li AR, et al. Epidermal growth factor receptor expression and gene amplification in colorectal carcinoma: an immunohistochemical and chromogenic in situ hybridization study. Mod Pathol 2005;18:1350-1356.

35 Dobashi Y, Shoji M, Jiang SX, et al. Active cyclin A-CDK2 complex, a possible critical factor for cell proliferation in human primary lung carcinomas. Am J Pathol 1998;153:963-972.

36 Han SW, Hwang PG, Chung DH, et al. Epidermal growth factor receptor (EGFR) downstream molecules as response predictive markers for gefitinib (Iressa, ZD1839) in chemotherapy-resistant non-small cell lung cancer. Int J Cancer 2005;113:109-115.

37 Kijima T, Niwa H, Steinman RA, et al. STAT3 activation abrogates growth factor dependence and contributes to head and neck squamous cell carcinoma tumor growth in vivo. Cell Growth Differ 2002;13:355-362.

38 Song JI, Grandis JR. STAT signaling in head and neck cancer. Oncogene 2000;19:2489-2495.

39 Dolloff NG, Shulby SS, Nelson AV, et al. Bonemetastatic potential of human prostate cancer cells correlates with Akt/PKB activation by alpha plateletderived growth factor receptor. Oncogene 2005;24: 6848-6854. 
40 Xia L, Wang L, Chung AS, et al. Identification of both positive and negative domains within the epidermal growth factor receptor $\mathrm{COOH}$-terminal region for signal transducer and activator of transcription (STAT) activation. J Biol Chem 2002;277: 30716-30723.

41 Shigematsu H, Lin L, Takahashi T, et al. Clinical and biological features associated with epidermal growth factor receptor gene mutations in lung cancers. J Natl Cancer Inst 2005;97:339-346.

42 Blaskovich MA, Sun J, Cantor A, et al. Discovery of JSI124 (cucurbitacin I), a selective Janus kinase/signal transducer and activator of transcription 3 signaling pathway inhibitor with potent antitumor activity against human and murine cancer cells in mice. Cancer Res 2003;63:1270-1279.

43 Mandal M, Kim S, Younes MN, et al. The Akt inhibitor KP372-1 suppresses Akt activity and cell proliferation and induces apoptosis in thyroid cancer cells. Br J Cancer 2005;92:1899-1905.

44 Kauffmann-Zeh A, Rodriguez-Viciana P, Ulrich E, et al. Suppression of c-Myc-induced apoptosis by Ras signalling through PI(3)K and PKB. Nature 1997; 385:544-548. 